\section{Hydrogel macht Prostatabestrahlung sicherer}

Die Herstellung eines Abstands zwischen Rektum und Prostata bei der intensitätsmodulierten Strahlentherapie (IMRT) des Prostatakarzinoms mit einem Zweikomponenten-Polyethylenglykol-Gel hatte in einer Phase-III-Studie nach 15 Monaten die Toxizität reduziert und die Lebensqualität verbessert. Jetzt liegen die Endergebnisse nach median 37 Monaten vor.

$\mathrm{n}$ der Studie randomisierten die Prüfärzte 222 Patienten mit niedrigem oder intermediärem Risiko im Verhältnis 2:1, entweder nur die Lokalisationsmarker oder zusätzlich das Gel zu erhalten. Alle Patienten wurden mit 79,2 Gy in 1,8-GyFraktionen unter Bildkontrolle bestrahlt. Ausgewertet wurden kumulative Toxizität und Lebensqualität (QoL) mit dem „Expanded Prostate Cancer Index Composite" (EPIC). Die Drei-Jahres-Inzidenz der rektalen Toxizität war im GelArm deutlich verringert. Toxizitäten vom Grad $\geq 1$ traten bei 9,2\% der Pati- enten ohne und bei 2,0\% mit Gel auf $(p=0,028)$, rektale Toxizitäten vom Grad $\geq 2$ bei $5,7 \%$ der Patienten ohne und bei keinem mit dem Gel. Auch die Harninkontinenzrate war ohne Verwendung des Gels deutlich höher (Grad $\geq 1$ : 15 vs. $4 \% ; \mathrm{p}=0,046$ ), allerdings ohne Unterschiede bei Harninkontinenz vom Grad $\geq 2$ (7 vs. $7 \% ; p=0,7$ ).

Nach drei Jahren hatten deutlich mehr Männer in der Kontrollgruppe im Vergleich zum Gel-Arm eine Abnahme der organspezifischen QoL erlitten (DarmQoL: 41 vs. $14 \%$; $\mathrm{p}=0,002$; Blase: 30 vs.
$17 \% ; p=0,04)$. Ein solcher Unterschied fand sich ebenfalls bezüglich einer starken Abnahme der Darm-QoL (21 vs. $5 \%$; $\mathrm{p}=0,02)$ und Blasen-QoL (23 vs. $8 \%$; $\mathrm{p}=0,02)$. In keinem der beiden Arme erlitt ein Patient einen erneuten PSA-Anstieg im Sinne eines biochemischen Therapieversagens. Der Abfall im PSA-Wert war in beiden Armen vergleichbar.

Fazit: Die Auswertung nach drei Jahren zeigt, dass der bereits belegte Vorteil des Zweikomponenten-PolyethylenglykolGels bezüglich der Toxizität bei der IMRT mit Bildkontrolle erhalten bleibt und sich mittelfristig günstig auf die organspezifische Lebensqualität auswirkt. Dabei scheint sich der Vorteil zugunsten des Gels zu vergrößern. Friederike Klein

Hamstra DA et al. Continued Benefit to Rectal Separation for Prostate Radiation Therapy: Final Results of a Phase III Trial. Int J Radiat Oncol Biol Phys. 2017;97(5):976-85.

\section{Chemotherapie beim Prostatakarzinom wechseln?}

\section{Spricht ein kastrationsresistentes Prostatakarzinom nicht frühzeitig auf eine} Therapie mit einem Taxan an, könnte der Wechsel zu einem anderen Taxan helfen. Das lassen Daten der Phase-II-Studie TAXYNERGY zumindest vermuten.

D arin erhielten 63 chemotherapienaive Patienten randomisiert im Verhältnis 2:1 Docetaxel bzw. Cabazitaxel. War nach dem vierten Zyklus der PSASpiegel noch nicht um $\geq 30 \%$ abgefallen, war ein Wechsel auf das jeweils andere Taxan vorgesehen. Als primärer Endpunkt wurde eine PSA-Abnahme $\geq 50 \%$ gegenüber historischen Kontrollen aus der TAX327-Studie definiert. Als Bio- marker für das Ansprechen wurden u. a. zu verschiedenen Zeitpunkten zirkulierende Tumorzellen (CTC) und die Abnahme des Androgenrezeptors im Nukleus (\%ARNL) bestimmt.

35 Patienten $(55,6 \%)$ erreichten unter dieser Therapie ein PSA-Ansprechen um $\geq 50 \%$ - deutlich mehr als bei den historischen Kontrollen (45,4\%). Von den 61 tatsächlich behandelten Patienten (zwei

\begin{tabular}{|c|c|c|c|}
\hline Switch-Status & Alle Patienten $(n=61)$ & Cabazitaxel $(n=22)$ & Docetaxel $(n=39)$ \\
\hline kein Taxan-Switch & $46(75,4 \%)$ & $19(86,4 \%)$ & $27(69,2 \%)$ \\
\hline kein Switch nach Zyklus 4 & $33(54,1 \%)$ & $13(59,1 \%)$ & $20(51,3 \%)$ \\
\hline Switch nicht umsetzbar* & $13(21,3 \%)$ & $6(27,3 \%)$ & $7(17,9 \%)$ \\
\hline Taxan-Switch & $15(24,6 \%)$ & $3(13,6 \%)$ & $12(30,8 \%)$ \\
\hline
\end{tabular}

im Docetaxel-Arm erhielten keine Therapie) erreichten 15 (24,6\%) den 30\%igen PSA-Abfall nicht und wechselten das Taxan. Bei sieben davon $(46,7 \%)$ nahm daraufhin das PSA um $\geq 50 \%$ ab.

Ein taxaninduzierter Abfall des \%ARNL von Tag 1-8 im ersten Zyklus war assoziiert mit einer höheren Rate von PSA-Abfällen $\geq 50 \%$ nach Zyklus $\operatorname{vier}(p=0,009)$. Das mediane progressionsfreie Überleben der Teilnehmer insgesamt lag zum Auswertungszeitpunkt bei 9,1 Monaten, der Median für das Gesamtüberleben war noch nicht erreicht.

Fazit: Trotz der wenigen Patienten mit Therapiewechsel (Tab.) sehen die Forscher bestätigt, dass die Switch-Strategie $\mathrm{zu}$ einem besseren PSA-Ansprechen führt. Bei Bestätigung in größeren Studien könnte zudem die taxanbedingte Veränderung des \%ARNL ein Biomarker sein, um frühzeitig Patienten zu erkennen, die von Taxanen profitieren.

Friederike Klein

Antonarakis ES et al. Randomized, Noncomparative, Phase II Trial of Early Switch From Docetaxel to Cabazitaxel or Vice Versa, With Integrated Biomarker Analysis, in Men With Chemotherapy-Naïve, Metastatic, Castration-Resistant Prostate Cancer. J Clin Oncol. 2017; 35 (28): 3181-8. 